\title{
Structure, dynamics and multiple length-scales in network-forming materials.
}

\author{
Mark Wilson \\ E-mail: mark.wilson@chem.ox.ac.uk \\ Department of Chemistry, Physical and Theoretical Chemistry Laboratory, \\ University of Oxford, South Parks Road, Oxford OX1 3QZ, U.K.
}

\begin{abstract}
Relationships between the structural and dynamical properties of network-forming materials are investigated. A generic model is utilised for systems of stoichiometry $\mathrm{MX}_{2}$ which are linked in the sense that they can all be usefully considered as constructed from linked $\mathrm{MX}_{4}$ tetrahedra. A single model parameter (the anion polarizability) is varied systematically to control the mean MXM bond angles (and hence the network topologies). The networks evolve from those dominated by cornersharing units to those dominated by edge-sharing structural motifs. These changes are accompanied by changes in the characteristic length-scales, with the emergence of ordering on intermediate length-scales. Key dynamical properties (the liquid relaxation just above the melting point and the liquid fragility) are studied and their relationship to the underlying static structure analysed.

PACS numbers: 61.20.Gy, 61.20.Ja, 61.20.Lc, 66.10.-x
\end{abstract}

\section{Introduction.}

As highlighted elsewhere in this special issue [1] potential links between structure and dynamics in the (disordered) liquid state is often (reasonably) reduced to a problem depending upon the nearest-neighbour interactions, effectively condensing the problem to the shortest length-scale which shows significant ordering (more often than not a packing effect). Many systems are, however, potentially more complex, displaying ordering, even in disordered states, on more than one length-scale. For example, a range of systems of stoichiometry $\mathrm{MX}_{2}$ (where $\mathrm{M}$ is a cation and $\mathrm{X}$ an anion - the focus of the work presented here) often show ordering on length-scales of the order of $5-10 \AA$. At first glance the bonding in these systems should be relatively simple to rationalise, being dominated by electrostatic interactions $[2,3,4,5]$. However, the 5$10 \AA$ length-scale is longer than that imposed by the simple charge electrostatics (anions surround cations etc - termed short-range order, SRO) and so is often referred to as intermediate-range order (IRO). The IRO is characterised by the presence of a so-called first-sharp diffraction peak (FSDP) in the total scattering function $[6,7,8]$ whose exact position may vary both as a function of the chemical nature of the system itself and the 
Structure, dynamics and multiple length-scales in network-forming materials.2

state point. Several systems of this stoichiometry have been investigated at a partial structure factor level; $\mathrm{ZnCl}_{2}\left({ }^{35,37} \mathrm{Cl}\right)[9,10,11], \mathrm{GeSe}_{2}\left({ }^{70,73} \mathrm{Ge},{ }^{N, 76} \mathrm{Se}\right)[10,12,13,14]$, $\mathrm{GeO}_{2}\left({ }^{70,73} \mathrm{Ge}\right)[15,16,17]$ and $\mathrm{SiO}_{2}[18]$.

Figure 1 highlights a range of systems of stoichiometry $\mathrm{MX}_{2}$ which can be considered as structurally linked in that, under ambient conditions, all can usefully be considered as constructed from $\mathrm{MX}_{4}$ tetrahedra. The primary differences between these systems lies in the manner in which these local coordination polyhedra (CP) are inter-linked. Two extremes can be identified. At one extreme each cation centre is connected to a neighbouring centre by a single anion "bridge" and hence a network is formed from corner-sharing tetrahedra only. At the other extreme a pair of cation centres may share two anion bridges (forming an edge-sharing unit). The presence of edge-sharing units has potentially significant implications for both structure and dynamics. For example, a percolating chain of edge-sharing units is charge neutral. As a result, an ion sufficiently removed from a given chain will not "see" the individual charges in that chain - they effectively cancel out. Consequently, systems of this sort (e.g. $\mathrm{SiSe}_{2}$ or $\mathrm{BeCl}_{2}$ ) can be considered as ensembles of polymeric chains despite maintaining dominant electrostatic interactions [19, 20, 21, 22]. Between these two extrema lie a rich complexity of network topologies. Systems such as $\mathrm{GeSe}_{2}[10,12,13,14,23,24,25,26,27,28,29]$ (and, perhaps, $\mathrm{ZnCl}_{2}$ [9, 10, 11, 30, 31, 32, 33, 34]) contain a mix of corner- and edge-sharing units (whose proportions may vary as a function of state point or, in the glassy state, as a function of preparation conditions). Furthermore, systems such as $\mathrm{BeF}_{2}, \mathrm{SiO}_{2}$ or $\mathrm{GeO}_{2}$, although formed exclusively from corner-sharing CP, may form very different network topologies, depending upon the connectivities of the constituent tetrahedra.

\section{Background.}

\subsection{Model.}

The potential employed are electrostatic polarizable-ion models (PIM) [35] which augment effective pair potentials (describing the short-range and dispersive interactions) and charge-charge interactions (a rigid-ion model - RIM) with a description of the (many-body) induced dipoles. The pair-potential elements of the models are parameterised in the standard manner (with respect to experimental data or utilising high level electronic structure calculations [36, 37]). The dipole polarization aspects are controlled by two key parameters; the anion polarizability $(\alpha)$ and the short-range damping parameter (SRDP). The anion polarizability controls the response of the ion electron density to the presence of an (external or internal) electric field. The SRDP controls the short-range (overlap) induced dipoles (which, for dipoles induced on anions act to oppose those induced by the formal ion charges) and may be parameterised directly from electronic structure calculations focussed on the (high symmetry) crystalline environments [38, 39, 40, 41]. 


\section{Structure, dynamics and multiple length-scales in network-forming materials.3}

\subsection{Methods.}

When utilising potential models of the type described above, two basic simulation strategies are employed:

- models are developed for specific systems of interest.

- generic models are developed in which (ideally) single parameters are varied in a systematic manner. For example, the anion polarizability in the PIM effectively controls the M-X-M (inter-tetrahedral) bond angle [42]

Each strategy has benefits and limitations. Building specific models for each system allows detailed comparison with experimental results and hence aids interpretation (see, for example, the analysis of the pressure-dependent evolution of structure in $\mathrm{SiO}_{2}[43]$ ). However, the simplifications and approximations required to develop models for different systems means that a number of key model parameters may vary from system to system (not necessarily in a systematic or physically transparent fashion). As a result, a detailed comparison between models is often problematic, at least in terms of identifying key parameters responsible for differing behaviour. Building generic models allows for a more direct comparison of the evolution of observable properties. However, detailed agreement with experimental data may be lost.

Structure factors are, in many ways, the most useful functions for studying density fluctuations over more than one length-scale. For example, these functions may display well-defined structure at low $k$ which corresponds to intermediate-range fluctuations in density and which may be directly measured. Ashcroft-Langreth (AL) structure factors are defined as [44], $S_{\alpha \beta}(k)=<A_{\alpha}^{*}(k) \cdot A_{\beta}(k)>$, where the Fourier components $A_{\alpha}(k)=\frac{1}{\sqrt{N_{\alpha}}} \sum_{i} e^{i \mathbf{k} \cdot \mathbf{r}_{i}}$ and $\left\{\mathbf{r}_{i}\right\}$ are the ion positions and $\alpha \beta$ correspond to the ion pairs. Alternatively, the Faber-Ziman (FZ) structure factors [44] $\left(S_{\alpha \beta}^{F Z}(k)\right)$ are defined relative to the Ashcroft-Langreth functions through the transformations [44, 45]

$$
S_{\alpha \beta}^{F Z}(k)=1+\frac{S_{\alpha \beta}(k)-\delta_{\alpha \beta}}{\left(c_{\alpha} c_{\beta}\right)^{1 / 2}},
$$

where $c_{\alpha}$ is the mole fraction of species $\alpha$. The choice of Ashcroft-Langreth or FaberZiman functions is arbitrary as they contain equivalent information regarding atom positions, differing only in their relative intensities. These structure factor definitions de-convolute the total structure factor (which could be obtained from a single diffraction experiment) in terms of the ion identities. The advantage is that the meaning of these functions is relatively clear in the sense that we "know where" the atoms of each type are in relation to one another. However, successful extraction from experimental data relies on an appropriate contrast between the respective atom scattering lengths.

Alternatively, in the Bhatia-Thornton (BT) formalism [46] the total structure factor is decomposed into functions depending on the chemical $(\mathrm{C})$ and topological $(\mathrm{N})$ ordering, resulting in three functions; $\mathrm{S}_{N N}(\mathrm{k}), \mathrm{S}_{N C}(\mathrm{k})$ and $\mathrm{S}_{C C}(\mathrm{k})$. The BT functions can then be generated from the Faber-Ziman functions using

$$
S_{C C}(k)=c_{M} c_{X}\left\{1+c_{M} c_{X}\left[\left(S_{M M}^{F Z}(k)-S_{M X}^{F Z}(k)\right)+\left(S_{X X}^{F Z}(k)-S_{M X}^{F Z}(k)\right)\right]\right\}
$$


Structure, dynamics and multiple length-scales in network-forming materials.4

$$
\begin{aligned}
& S_{N N}(k)=c_{M}^{2} S_{M M}^{F Z}(k)+c_{X}^{2} S_{X X}^{F Z}(k)+2 c_{M} c_{X} S_{M X}^{F Z}(k) \\
& S_{N C}(k)=c_{M} c_{X}\left\{c_{M}\left[S_{M M}^{F Z}(k)-S_{M X}^{F Z}(k)\right]-c_{X}\left[S_{X X}^{F Z}(k)-S_{M X}^{F Z}(k)\right]\right\},
\end{aligned}
$$

or from the Ashcroft-Langreth functions using

$$
\begin{aligned}
& S_{C C}(k) /\left(c_{M} c_{X}\right)=c_{X} S_{M M}(k)+c_{M} S_{X X}(k)-2 \sqrt{c_{M} c_{X}} S_{M X}(k) \\
& S_{N N}(k)=c_{M} S_{M M}(k)+c_{X} S_{X X}(k)+2 \sqrt{c_{M} c_{X}} S_{M X}(k) \\
& S_{N C}(k) /\left(c_{M} c_{X}\right)=S_{M M}(k)-S_{X X}(k)-\left(c_{M} c_{X}\right)^{-1 / 2}\left(c_{M}-c_{X}\right) S_{M X}(k) .
\end{aligned}
$$

The BT functions are potentially useful as they contain topological information not coloured by atom type. For example, $S_{C C}(k)$ is related to the charge-charge structure factor, $S_{Z Z}(k)$, and so contains information as to the length-scale over which local charge-neutrality is maintained. Experimentally each set of three partial structure factors can be generated from the total structure factors using different isotopic compositions. Significantly, the different weightings in the contributions of the AL (or FZ) functions, with respect to the BT, to the total scattering function may mean extracting one or the other is preferable purely for numerical reasons. In simulation studies, however, the partial structure factors are generated directly from the ion positions (most simply for the Ashcroft-Langreth functions described above), which may be then transformed into the Faber-Ziman or Bhatia-Thornton) functions using the above equations.

\section{Results.}

\subsection{Static Structure.}

Figure 2 shows the evolution of the Ashcroft-Langreth partial structure factors as a function of anion polarizability, $\alpha$. The changes in $S_{M X}(k)$ and $S_{X X}(k)$ appear relatively minor with the principle peak positions (at $k_{P P} \sim 2.0 \AA^{-1}$ ) almost invariant with $\alpha$. The most dramatic changes are observed in $S_{M M}(k)$. At low $\alpha$ (corresponding to a RIM) $S_{M M}(k)$ appears as a single peak at $k_{P P} \sim 1.5 \AA^{-1}$ with a significant "shoulder" at higher $k$. As the polarizability increases this peak "splits" into a principle peak (at $k_{P P} \sim 2.0 \AA^{-1}$ ) and a $\operatorname{FDSP}\left(\right.$ at $k_{F S D P} \sim 1.0 \AA^{-1}$ ). The inset to figure 2 (c) highlights that the position of the FSDP effectively "saturates" at high $\alpha$. The behaviour of $S_{M M}(k)$ as a function of $\alpha$ is relatively simple to rationalise. At low $\alpha$ (corresponding to a RIM) the charge-charge electrostatic interactions dominate the structure and so the nearest-neighbour cation-cation length-scale is typically relatively long (reflecting the higher cation formal charges). This relatively long length-scale corresponds to the peak in $S_{M M}(k)$ at relatively low $k$. Figure 3 shows the M-X$\mathrm{M}$ bond angle distributions as a function of $\alpha$. As the polarizability increases the mean MXM bond angle is reduced (corresponding to falls in the M-M nearest-neighbour separations). Dipoles induced on the bridging anions act to introduce negative charge to in-between neighbours of cation pairs and, as a result, effectively screens the chargecharge interaction [35]. The reduction of the nearest-neighbour cation-cation length- 


\section{Structure, dynamics and multiple length-scales in network-forming materials.5}

scale leads to a depletion of cation density on an intermediate length-scale and hence two peaks in the structure factor emerge; the principle peak corresponding to the emergence of density fluctuations on the short length-scale, and the first-sharp diffraction peak emerging from the corresponding depletion of cation density on a longer length-scale $[47,48]$.

Figure 4 shows the Bhatia-Thornton structure factors as a function of anion polarizability. $S_{N N}(k)$ shows a "three peak" structure identified as typical of networkforming melts $[49,50]$ and showing a FSDP across all anion polarizabilities. For $S_{N C}(k)$ the FSDP is concurrence with that in $S_{N N}(k)$ [49]. Interestingly, the intensity of the FSDP in $S_{C C}(k)$ appears to increase with $\alpha$. Neutron diffraction studies show the presence of an FSDP in $S_{C C}(k)$ in $\mathrm{GeSe}_{2}$ and, to a lesser extent, in $\mathrm{ZnCl}_{2}$ [10]. However, the presence of an FSDP in $S_{C C}(k)$ remains somewhat controversial [42], although there is no formal requirement for the FSDP to be suppressed. In a rigid-ion (charge-only) system $S_{C C}(k)$ is related to the charge-charge structure factor, $S_{Z Z}(k)$, by $S_{Z Z}(k)=$ $\left(c_{A} c_{B}\right)^{-1} S_{C C}(k)$. For overall electroneutrality the sum of all charges must be zero over all space (the Stillinger-Lovett first sum rule). A charge imbalance on an intermediate length-scale appears perfectly permissible. Interestingly, different models and methods produce differing results for the intensity of $S_{C C}\left(k_{F S D P}\right)$. Potential models for $\mathrm{GeSe}_{2}$ which use explicit three-body (bond-bending) terms show no clear FSDP $[51,52,53,54]$. However, closer inspection indicates that the structures generated appear more $\mathrm{GeO}_{2}{ }^{-}$ like in being more charge-ordered and, as a result, equate with a low $\alpha$ function on figure $4(\mathrm{c})$ which show a hint of low $k$ shoulder on the principle peak. Density-functional-based electronic structure calculations using a non-self-consistent method shows a clear FSDP $[55,56,57,58]$. However, calculations utilising both local and gradient-corrected density approximations show no such feature $[23,24,25,26,27,28]$. PIM simulations for $\mathrm{ZnCl}_{2}$ [59] and $\mathrm{GeSe}_{2}$ [60] relate the intensity of $S_{C C}\left(k_{F S D P}\right)$ to the fraction of edge-sharing units [56]. A possible explanation, therefore, is that different levels of density-functional approximation controls the (fine) balance between the relative stabilities of the cornerand edge-sharing units, and hence controls the intensity of any FSDP in $S_{C C}(k)$.

Figure 5 (left panel) shows the partial pair distribution functions extracted at four different anion polarizabilities compared (right panel) to the analogous data obtained from neutron diffraction experiments using isotopic substitution [15, 16, 10, 11, 14, $13,12,13]$. Successive functions are offset along the ordinate axis for clarity. The functions are coloured as in panel (a). The experimental data is arranged to mimic the series highlighted in figure 1 . The data is scaled by the nearest-neighbour anioncation separation (in all cases) which is equivalent to scaling in terms of the length-scale imposed by the tetrahedral CP. The key changes are observed in the cation-cation partial pair distribution functions. For the simulation results the mean M-M bond length falls as the anion polarizability increases (reflecting, naturally, the bond angle distributions in figure 3). For larger polarizabilities $g_{M M}(r)$ shows two clear contributions to the first peak, corresponding to the presence of both corner- and edge-sharing tetrahedra, the latter becoming predominant at the highest polarizabilities. For the experimental 


\section{Structure, dynamics and multiple length-scales in network-forming materials.6}

results the scaled $g_{M M}(r)$ first peak shifts to low $r$ moving along the series indicated in figure 1. The peak obtained from liquid $\mathrm{GeSe}_{2}$ shows a hint of a "two-peak" (corner- and edge-sharing) structure observed in simulation. The glassy and liquid $\mathrm{GeSe}_{2} g_{M M}(r)$ functions show significant differences, hinting at the edge-sharing units as transient intermediates rather than energetically favourable local structures.

To further characterise the corner- and edge-sharing units their connectivities can be broken down into three types; '0' - all links are corner-sharing, ' 1 ' - two of the links are corner-sharing, one in edge-sharing, and ' 2 ' - both links are edge-sharing $[23,24,25,26,27,28]$. Figure 3(b) shows the evolution of these species as a function of anion polarizability. As the polarizabilty increases the fraction of ' 1 ' and ' 2 ' sites increases (corresponding to an increase in the number of edge-sharing units). The "saturation" of the FSDP in $S_{M M}(k)$ in figure 2 is strongly correlated with the emergence of edge-sharing units as the dominant structural motif. A "limiting" structure comprised purely of these units has an essentially polymeric structure (as the edge-sharing chains are charge neutral). The chain-chain length-scale (which now dominates $S_{M M}\left(k_{F S D P}\right)$ ) is now essentially fixed by the sizes of the atoms. Any further increase in anion polarizability may compress the chains (lowering the nearest-neighbour M-M distance and distorting the tetrahedra) but will have little effect of the inter-chain separation (and hence the FSDP location).

\subsection{Dynamics.}

The figures in the previous section highlight the evolution of network structure (and hence topology) as the bond angles between neighbouring tetrahedral units are varied in a systematic manner. What is unclear from this analysis is how these changes affect the system dynamics.

3.2.1. Relaxation. Figure 6 shows the intermediate scattering functions, $F_{M M}\left(k_{X}, t\right)$, calculated along the $T=800 \mathrm{~K}$ isotherm (just above the respective melting points) at two polarizabilities; $\alpha=15 \mathrm{au}$ and 25au, corresponding to regimes in which the corner- and edge-sharing units dominate respectively. In both cases these functions are calculated at scattering angles which correspond to the positions of the respective principle $(\mathrm{X}=\mathrm{PP})$ and first-sharp diffraction $(\mathrm{X}=\mathrm{FSDP})$ peaks. In all cases the functions show characteristic short-time $(\beta)$ and longer-time $(\alpha)$ relaxation. The $\beta$-relaxation regime is characteristic of the motion of the ions within their nearest-neighbour "cages" and, as a result, appears significantly stronger in the principal peaks (which correspond to the SRO). The $\alpha$-relaxation functions are fitted to stretched exponential functions of the form $F_{M M}^{f i t}\left(k_{X}, t\right)=A \exp \left[(-t / \tau)^{\gamma}\right]$. For the $\alpha=25$ au model the time-scale for $\alpha$-relaxation on the length-scale associated with the FSDP is now considerably longer than that associated with the principle peak. As the system is cooled, therefore, it is the density fluctuations on the intermediate length-scale which control the vitrification dynamics in contradiction to systems which arrest at the glass transition temperature 
Structure, dynamics and multiple length-scales in network-forming materials.7

due to pure packing effects.

3.2.2. Diffusion. Figure 7 shows the self-diffusion coefficients, $D$, calculated for the cations from the mean-squared displacements as a function of temperature, $T$. The diffusion coefficients are calculated over a range of anion polarizabilities and are shown at two densities. The data are plotted as $\ln D v .1 / T$ in order to identify any curvature, characteristic of the relative fragility of the liquid as cooled towards the glass transition temperature. To quantify this fragility data were fitted to a Vogel-Fulcher-Tammann (VFT) law of the form $D=D_{0} \exp \left[-B /\left(T-T_{0}\right)\right]$ where $B$ and $T_{0}$ control curvature and hence characterize the fragility $[61,62]$ as highlighted by the $T_{0} / B$ ratios which are shown as insets. The VFT functions were used to generate effective glass transition temperatures, $T_{f}$, corresponding to $\ln \left(D / D_{0}\right)=-10$, which were used to scale the temperature axis in figure 7 [61]. At both densities, the fragility increases for $\alpha>15$ au. At higher density an Arrhenius temperature dependence $\left(T_{0} / B=0\right)$ is observed for $\alpha=15$ au. The clear implication, therefore, is that the fraction of edge-sharing species effectively correlates with the system fragility. Systems with high fractions of edgesharing units tend towards behaving as a relatively fragile pseudo-polymeric ensemble of chains (analogous to relatively simple organic molecular systems which would be expected to be relatively fragile).

3.2.3. Topology. In order to probe the dynamic evolution of the network topology, two approaches are considered.

- Time correlation functions are constructed which monitor the life-times of specified cation environments.

- Van Hove spatial correlation functions are constructed which are "coloured" by cation environment.

The time correlation function is defined as

$$
f(t)=\frac{1}{N_{\text {cat }}} \sum_{i=1}^{N_{\text {cat }}} n_{i}(t) \cdot n_{i}(0),
$$

where $n_{i}(t)$ is the label " 0 ", "1" or "2" (defining the corner- and edge-sharing environment as described earlier) for cation $i$. The product $n_{i}(t) \cdot n_{i}(0)$ is defined as

$$
\begin{aligned}
n_{i}(t) \cdot n_{i}(0)=1 & \text { if } \quad n_{i}(t)=n_{i}(0) \\
=0 & \text { if } \quad n_{i}(t) \neq n_{i}(0) .
\end{aligned}
$$

Figure 8 shows $f(t)$ for $n_{i}=2,1$ and 0 respectively for $\alpha=22.5$ au at five temperatures $(T=1500,1200,1000,900$ and $800 \mathrm{~K})$. This system is chosen as an example of one in which all three environments are present in significant quantities. At high temperature all three functions are observed to decay over similar time-scales, indicating that the effective "residence times" of the three labelled cation environments to be approximately equal. However, as the system is cooled the time-scales over which the three functions decay separate. Those species labelled "2" decay more slowly than those labelled "1" 


\section{Structure, dynamics and multiple length-scales in network-forming materials.8}

which, in turn, decay more slowly than those labelled "0". At temperatures near the melting point, therefore, the effective residence time for the edge-sharing units is significantly longer than for the corner-sharing.

The (distinct) van Hove correlation function is defined by

$$
G_{\alpha \beta}\left(\mathbf{r}^{\prime}, \mathbf{r}, t\right)=\left\langle\rho_{M}^{\alpha}(\mathbf{r}, 0) \cdot \rho_{M}^{\beta}\left(\mathbf{r}^{\prime}, t\right)\right\rangle,
$$

where $\rho_{M}^{\alpha}(\mathbf{r}, 0)=\frac{1}{N_{\alpha}} \sum_{i=1}^{N_{\alpha}} \delta\left[\mathbf{r}-\mathbf{r}_{i}(t)\right]$ and $\alpha$ and $\beta$ refer to the labels "2", "1" or "0" as described previously. The van Hove correlation function contains information as to how the inter-atomic arrangements evolve with time. They may be divided into "self" and "distinct" terms, the former containing information as to how atom locations evolve relative to the same atoms original locations, and the latter containing information as to how the correlations between different atoms evolve. At short times, for example, the distinct terms will look like the respective static pair distribution functions but, as time increases, will become less structured reflecting the averaging out associated with diffusive motion. In this case the definition leads to six functions which highlight the spatial evolution of one coloured site with respect to another. Figure 9 shows the evolution of these six van Hove correlation functions generated along the $T=1000 \mathrm{~K}$ isotherm. The first peak positions vary, reflecting the longer length-scale associated with corner-, compared with edge-, sharing units. For example, a "2" unit will correspond to a relatively acute M-X-M bond angle and hence a relatively short M-M separation. The absence of a first peak in $G_{02}(r, t)$ reflects the fact that a cation labelled ' 0 ' cannot connect directly to one labelled ' 2 '. The feature at $r \sim 6 \AA$ corresponds to the lengthscale associated with the FSDP and appears relatively stable. Significantly, this feature appears more prominent in the functions which incorporate edge-sharing units. The relative prominence increases as the dynamics evolves.

The time- and van Hove-correlation functions highlight the role of corner- and edgesharing units in controlling the underlying dynamics in these systems. Edge-sharing units appear more stable (in a thermodynamic sense) and contribute more strongly to ordering on the intermediate length-scale. As discussed above the latter can be rationalised by considering a system comprised entirely from edge-sharing units. In the $\mathrm{MX}_{2}$ stoichiometry the system then becomes a polymeric network of edge-sharing chains and, as a result, the intermediate-range order becomes dominated by the interchain spatial correlations.

\subsection{Structure and Dynamics.}

The results described here show how the underlying static structure of the $\mathrm{MX}_{2}$ systems (dominated by $\mathrm{MX}_{4}$ tetrahedra) can be rationalised. In an electrostatics-driven model (anions and cations) it is the anion polarizability which controls the M-X-M bond angles and, as a result, controls the spatial relationship between neighbouring tetrahedral units and, as a result, controls the network topology. As the M-X-M angle is lowered an intermediate-range length-scale emerges which, critically, is found to dominate the low temperature liquid relaxation dynamics. As these bond angles become yet more 
Structure, dynamics and multiple length-scales in network-forming materials.9

acute edge-sharing units become stable, which is important as these units percolate to generate charge neutral chains which significantly alter the dominant interactions. This change transfers to a consideration of the dynamics. The fraction of edge-sharing units affects the liquid fragility. In a simplistic sense the system evolves from a "strong" three-dimensional corner-sharing $\left(\mathrm{SiO}_{2}\right.$-like) network to a "fragile" structure based on a polymeric structure of chains of edge-sharing tetrahedra. Furthermore, the edge-sharing units start to control the ordering on the intermediate length-scale as they are longerlived than the corner-sharing units.

\section{Conclusions.}

The network-forming systems considered here are related in the sense that their structures can be rationalised in terms of inter-linked tetrahedral local coordination

polyhedra. The topology of these networks can be systematically modified using a generic model, and this allows the evolution of ordering on multiple length-scales to be observed and rationalised. The emergence of intermediate-range order is found to control the liquid relaxation dynamics at the glass transition temperature is approached during cooling. The formation of edge-sharing tetrahedral units, which are found to be relatively stable (long-lived) species, has a significant effect on the liquid fragility.

\section{Acknowledgements.}

It is a pleasure to acknowledge Professor Philip Salmon and Dr. Anita Zeidler (both in the Department of Physics, University of Bath, U.K.) for sharing experimental data and for many helpful and fruitful discussions.

[1] This issue. J. Stat. Mech.

[2] J.-P. Hansen and I.R. McDonald. Theory of Simple Liquids. Academic Press, New York, 1986.

[3] A.R.Ubbelohde. The Molten State of Matter. John Wiley and Sons, 1978.

[4] M.Rovere and M.P.Tosi. Rep. Prog. Phys, 49:1001, 1986.

[5] N.H. March and M.P.Tosi. Coulomb Liquids. Academic Press, 1984.

[6] S.R.Elliott. Nature, 354:445, 1991.

[7] P.S.Salmon. Proc. R. Soc. Lond. A, 445:351, 1994.

[8] P. S. Salmon and A. Zeidler. Phys. Chem. Chem. Phys., 15:15286, 2013.

[9] S.Biggin and J.E.Enderby. J. Phys. C, 14:3129, 1981.

[10] P.S.Salmon, R.A.Martin, P.E.Mason, and G.J.Cuello. Nature, 435:75, 2005.

[11] A.Zeidler, P.S.Salmon, S.Philip, R.A.Martin, T.Usuki, P.E.Mason, G.J.Cuello, S.Kohara, and H.E.Fischer. Phys. Rev. B, 82:104208, 2010.

[12] I.Petri, P.S.Salmon, and H.E.Fischer. Phys. Rev. Lett., 84:2413, 2000.

[13] P.S.Salmon and I.Petri. J. Phys.: Condens. Matter, 15:S1509, 2003.

[14] I.T.Penfold and P.S.Salmon. Phys. Rev. Lett., 67:97, 1991.

[15] P.S.Salmon, A.C.Barnes, R.A.Martin, and G.J.Cuello. Phys. Rev. Lett., 96:235502, 2006.

[16] P.S.Salmon, A.C.Barnes, R.A.Martin, and G.J.Cuello. J. Phys.: Condens. Mat., 19:415110, 2007.

[17] K. Wezka, P. S. Salmon, A. Zeidler, D. A. J. Whittaker, J. W. E. Drewitt, S. Klotz, H. E. Fischer, and D. Marrocchelli. J. Phys.: Condens. Mat., 24:502101, 2012.

[18] Q.Mei, C.J.Benmore, S.Sen, R.Sharma, and J.L.Yarger. Phys. Rev. B, 78:144204, 2008.

[19] V.D.Prisyazhnyi and S.P.Baranov. Zh. Neorg. Khim., 23:1678, 1978. 
Structure, dynamics and multiple length-scales in network-forming materials.10

[20] E.A.Pavlatou and V.I.Constadaras. unpublished.

[21] M. Wilson and P.A. Madden. Mol. Phys., 92:197, 1997.

[22] M. Wilson and M.C.C. Ribeiro. Mol. Phys., 96:867, 1999.

[23] C.Massobrio, F.H.M van Roon, A.Pasquarello, and S.W.De Leeuw. J. Phys.: Condens. Matt., 12:L697, 2000.

[24] C.Massobrio, M.Celino, and A.Pasquarello. Phys.Rev.B, 70:174202, 2004.

[25] C.Massobrio, A.Pasquarello, and R.Car. Phys.Rev.Lett., 80:2342, 1998.

[26] C.Massobrio, A.Pasquarello, and R.Car. Phys.Rev.B, 64:144205, 2001.

[27] C.Massobrio and A.Pasquarello. J.Chem.Phys., 114:7976, 2001.

[28] C.Massobrio and A.Pasquarello. Phys.Rev.B, 68:020201, 2003.

[29] K. Wezka, A. Bouzid, K. J. Pizzey, P. S. Salmon, A. Zeidler, S. Klotz, H. E. Fischer, C. L. Bull, M. G. Tucker, M. Boero, S. Le Roux, C. Tugne, and C. Massobrio. Phys. Rev. B, 90:054206, 2014 .

[30] J. Neuefeind and K.-D. Liss. Ber. Bunsenges. Phys. Chem., 100:1341, 1996.

[31] J. Neuefeind. Phys.Chem.Chem.Phys., 3:3987, 2001.

[32] A.Lemke J.Neuefeind, K.Toedheide and H.Bertagnolli. J.Non-Cryst. Solids, 224:205, 1998.

[33] A.Zeidler, P.Chirawatkul, P.S.Salmon, T.Usuki, S.Kohara, H.E.Fischer, and W. S. Howells. J. Non-Cryst. Solids, 407:235, 2015.

[34] J. Neuefeind. arxiv.org/pdf/cond-mat/000613\%.

[35] P.A.Madden and M. Wilson. Chem. Soc. Rev., 25:339, 1996.

[36] A.Aguado, L.Bernasconi, and P.A.Madden. Chem. Phys. Lett., 356:437, 2002.

[37] A.Aguado, L.Bernasconi, S.Jahn, and P.A.Madden. Faraday Discuss., 124:171, 2003.

[38] P.W.Fowler and P.A.Madden. Phys. Rev. B, 31:5443, 1985.

[39] P. Jemmer, P.W.Fowler, M. Wilson, and P.A.Madden. J. Chem. Phys., 111:2038, 1999.

[40] C. Domene, P.W.Fowler, P.A.Madden, M. Wilson, and R.J.Wheatley. Chem. Phys. Lett., 333:403, 2001.

[41] C. Domene, P.W.Fowler, P.A.Madden, Jijun Xu, R.J.Wheatley, and M.Wilson. J. Phys. Chem. A, 105:4136, 2001.

[42] M.Wilson and B.K.Sharma. J.Chem.Phys., 128:214507, 2008.

[43] A.Zeidler, K.Wezka, D.A.J.Whittaker, P.S.Salmon, S.Klotz, H.E.Fischer, M.C.Wilding, C.L.Bull, M.G.Tucker, and M.Wilson. Phys. Rev. Lett., 113:135501, 2014.

[44] N. E. Cusack. The Physics of Structurally Disordered Matter. Hilger, Bristol, 1987, 1987.

[45] H.E.Fischer, A.C.Barnes, and P.S.Salmon. Rep. Prog. Phys., 69:233, 2006.

[46] A.B.Bhatia and D.E.Thornton. Phys.Rev.B, 2:3004, 1970.

[47] M.Wilson. J. Phys: Condensed. Matter, 24:284114, 2012.

[48] M.Wilson. Physical Chemistry Chemical Physics, 14:12701, 2012.

[49] P.S.Salmon. Proc: Maths and Phy. Sci., 437:591, 1992.

[50] A.C.Wright, R.N.Sinclair, and A.J.Leadbetter. J. Non-Cryst. Solids, 71:295, 1985.

[51] P.Vashishta, R.K.Kalia, and I.Ebbsjo. Phys. Rev. B, 39:6034, 1989.

[52] P.Vashishta, R.K.Kalia, G.A.Antonio, and I.Ebbsjo. Phys. Rev. Lett., 62:1651, 1989.

[53] P.Vashishta, R.K.Kalia, J.P.Rino, and I.Ebbsjo. Phys. Rev. B, 41:12197, 1990.

[54] H.Iyetomi, P.Vashishta, and R.K.Kalia. Phys. Rev. B, 43:1726, 1991.

[55] M.Cobb, D.A.Drabold, and R.L.Cappelletti. Phys.Rev.B, 54:12162, 1996.

[56] M.Cobb and D.A.Drabold. Phys.Rev.B, 56:3054, 1997.

[57] M.Durandurdu and D.A.Drabold. Phys.Rev.B, 65:104208, 2002.

[58] P.Biswas, D.N.Tafen, and D.A.Drabold. Phys.Rev.B, 71:054204, 2005.

[59] B.K. Sharma and M. Wilson. J. Phys.: Condens. Matter, 20:244123, 2008.

[60] R.Sharma, S.N.Chakraborty, and C.Chakravarty. J. Chem. Phys., 125:204501, 2006.

[61] S. Sastry. Nature, 409:164, 2001.

[62] K.Binder and W.Kob. Glassy Materials and Disordered Solids. World Scientific, Singapore, 2005. 


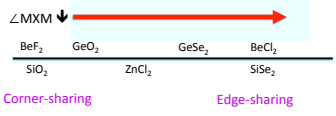

Figure 1. Schematic to show how the MXM ( $M=$ cation, $\mathrm{X}=$ anion) mean bond angles evolve for a range of related systems. The MXM bond angle falls from left to right, correlated with the evolution of network topologies from those dominated by cornersharing units to those dominated by edge-sharing units. 

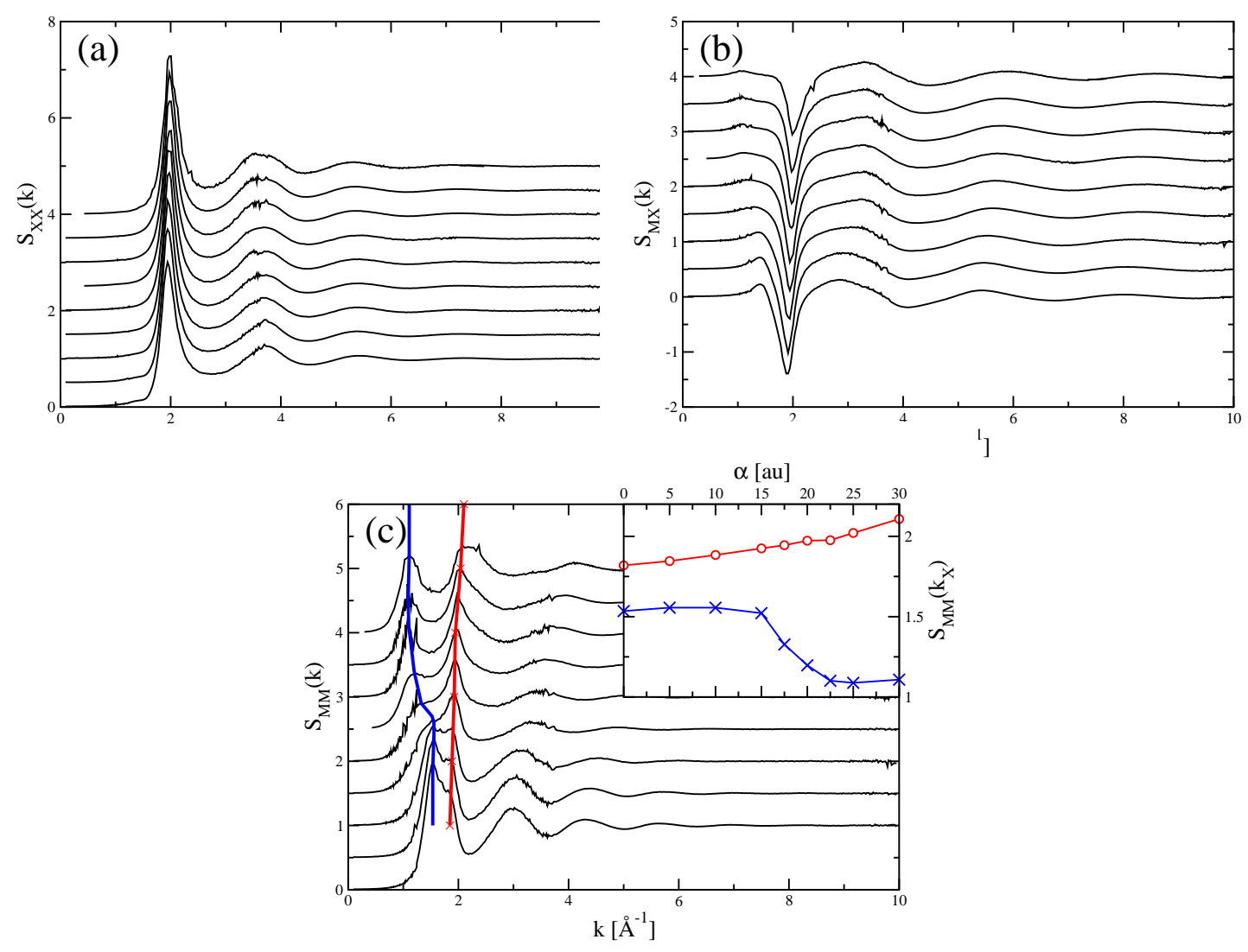

Figure 2. Ashcroft-Langreth partial structure factors for liquid $\mathrm{MX}_{2}$ (calculated along the $T=1000 \mathrm{~K}$ isotherm) as a function of anion polarizability, $\alpha$. The functions shown are (a) $S_{X X}(k)$, (b) $S_{M X}(k)$, (c) $S_{M M}(k)$. Successive functions are offset along the ordinate axis for clarity and, in each panel, correspond to (from bottom to top) $\alpha=0$, $5,10,15,17.5,20,22.5,25$ and 30au respectively. In the main panel of (c) lines are superimposed to highlight the evolution of the first two peak positions (which evolve to form the principle and first-sharp diffraction peaks [PP and FSDP] at high $\alpha$ ). The inset to (c) shows these two peak positions (X=PP or FSDP) as a function of $\alpha$. 
Structure, dvnamics and multiple length-scales in network-forming materials.13
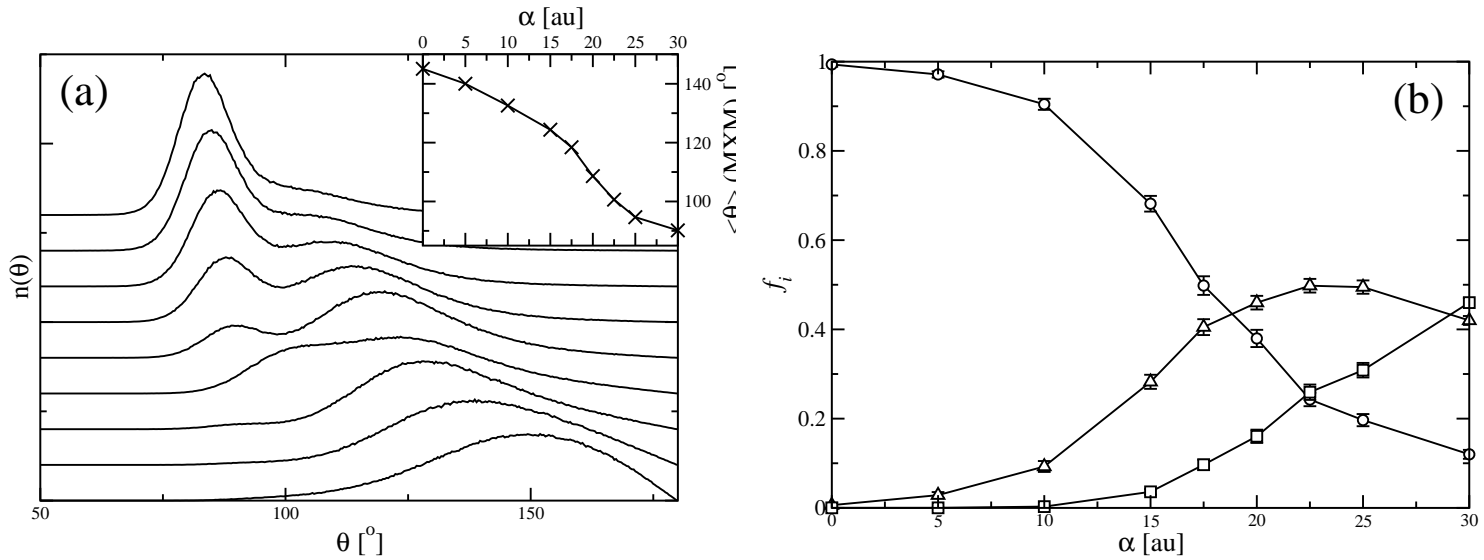

Figure 3. (a) M-X-M bond angle distributions shown as a function of anion polarizability, $\alpha$. Successive functions are offset along the ordinate axis for clarity. The functions shown are (from bottom to top) for $\alpha=0,5,10,15,17.5,20,22.5,25$ and 30au respectively. The inset shows the mean MXM bond angle as a function of $\alpha$. (b) Fractions of cation sites labelled "0" (circles), "1" (triangles) or "2" (squares) as a function of $\alpha$.
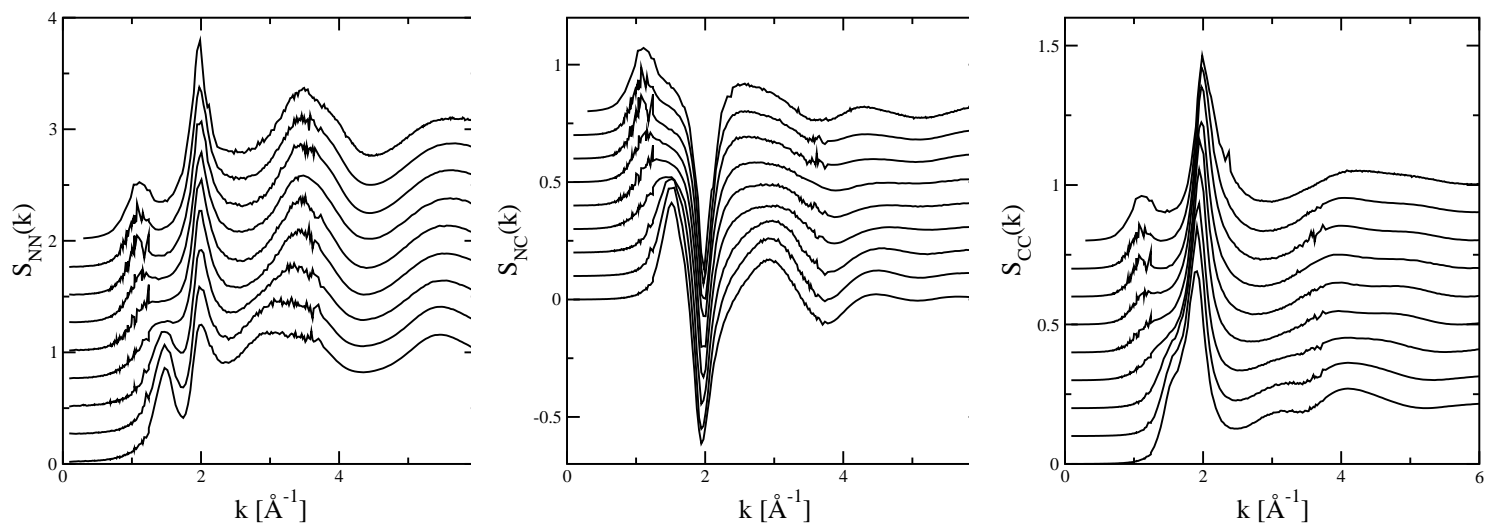

Figure 4. Bhatia-Thornton partial structure factors for liquid $\mathrm{MX}_{2}$ (calculated along the $T=1000 \mathrm{~K}$ isotherm) as a function of anion polarizability, $\alpha$. The functions shown are (a) $S_{N N}(k)$, (b) $S_{N C}(k)$, (c) $S_{C C}(k)$. Successive functions are offset along the ordinate axis for clarity and, in each panel, correspond to (from bottom to top) $\alpha=0$, $5,10,15,17.5,20,22.5,25$ and 30 au respectively. 


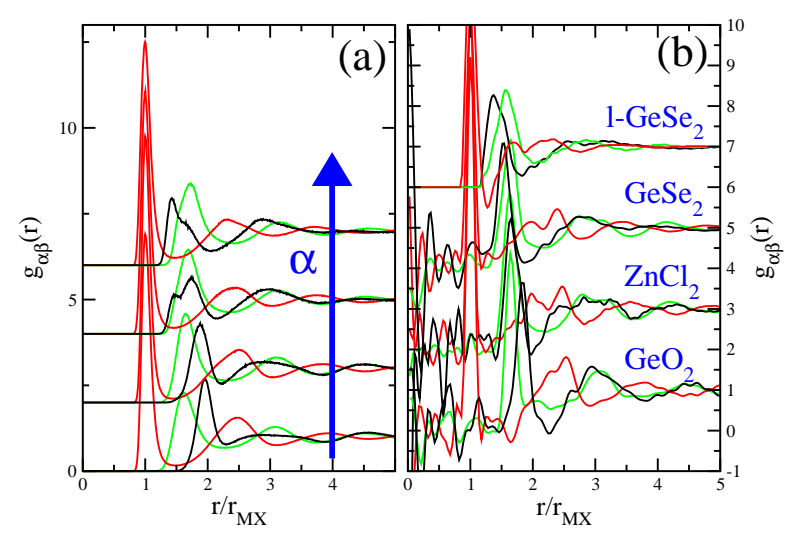

Figure 5. (a) The evolution of the pair distribution functions as a function of anion polarizability, $\alpha$. Key: red $-g_{M X}(r)$, green $-g_{X X}(r)$, black $-g_{M M}(r)$. Successive functions are offset along the ordinate axis for clarity. The functions shown are for (from bottom to top) $\alpha=0,10,20$ and 30au respectively. (b) Pair distribution functions obtained experimentally by neutron diffraction using isotopic substitution for $\mathrm{GeO}_{2}[15,16], \mathrm{ZnCl}_{2}[10,11]$ and $\mathrm{GeSe}_{2}$ in the liquid $[14,13]$ and glassy $[12,13]$ states. Successive functions are offset along the ordinate axis for clarity. The functions are coloured as in panel (a).
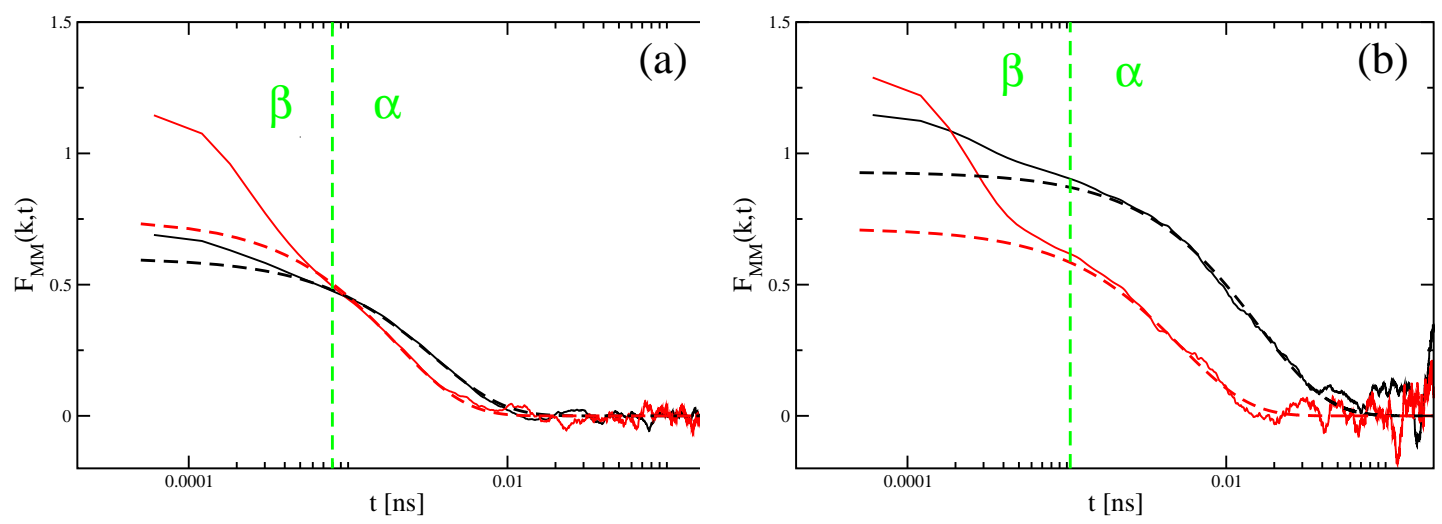

Figure 6. Intermediate scattering functions calculated for the cations, $F_{M M}(k, t)$, at $T=800 \mathrm{~K}$ for (a) $\alpha=15 \mathrm{au}$ and (b) $\alpha=25 \mathrm{au}$. In both cases the functions are shown for scattering angles which correspond to the positions of the respective first-sharp diffraction peaks (black lines) and principle peaks (red lines). The approximate $\alpha-$ and $\beta$ - relaxation regimes are indicated. The dashed lines show the results of fitting the $\alpha$-relaxation regime to stretched exponential functions. 

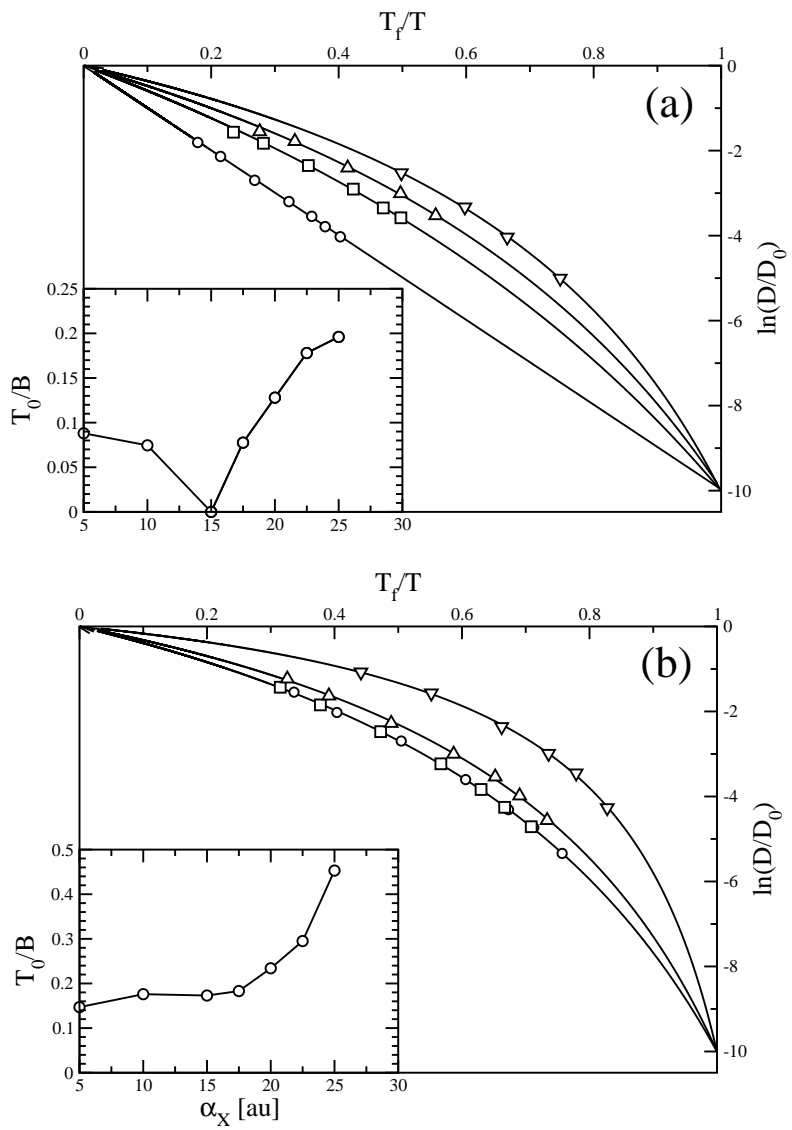

Figure 7. Cation diffusion coefficients, $D$, as a function of $T$ calculated over a range of anion polarizabilites, $\alpha$, and shows as $\ln D v .1 / T$ in order to highlight the respective curvatures (which relate to the liquid fragility). The diffusion coefficients are generated at two densities (shown in (a) and (b), panel (a) corresponding to the higher density). The diffusion coefficients are fitted to a VTF function as described in the text. In both cases the insets show the VTF fitting parameters, $T_{0} / B$, as a function of $\alpha$, highlighting the increase in svstem fragilitv as $\alpha$ increases.

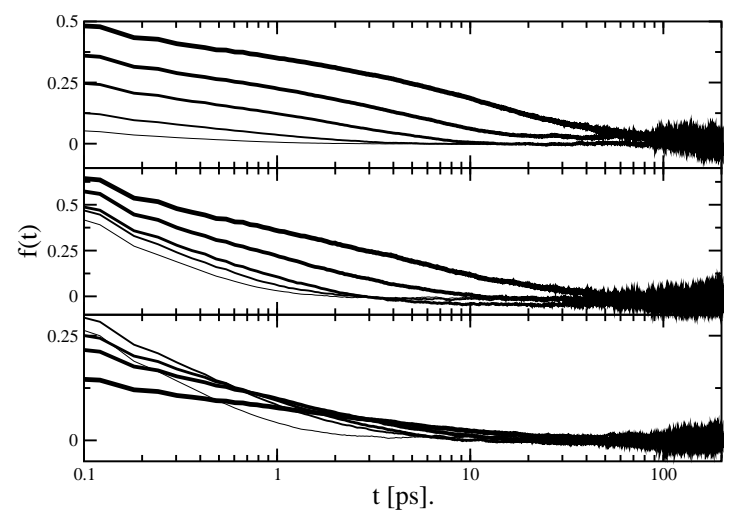

Figure 8. Time correlation functions constructed from the cation sites "coloured" by their connectivity as described in the text. The upper, central and lower panels show the respective decays of the functions for sites labelled ' 2 ', ' 1 ' and ' 0 ' determined at different temperatures (in order of increasing thickness, $T=$ 1500, 1200, 1000, 900and800 Krespectively. 


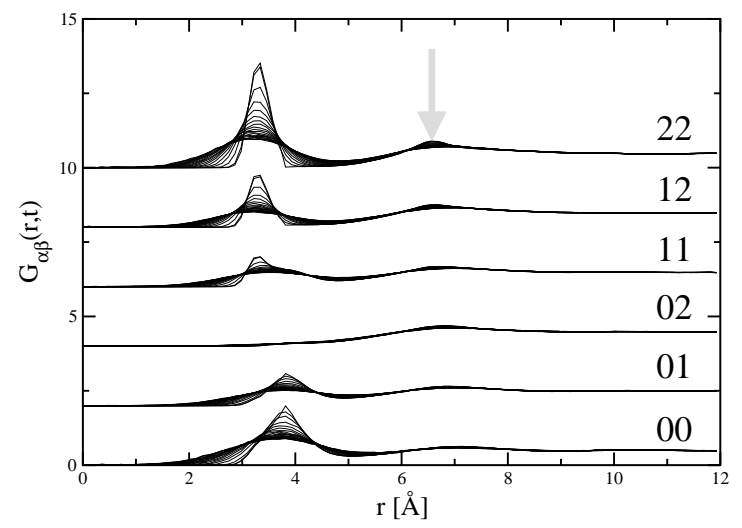

Figure 9. Distinct van Hove spatial correlation functions $G_{\alpha \beta}\left(\mathbf{r}^{\prime}, \mathbf{r}, t\right)$ obtained from the local cation environments $(\alpha \beta=\{00,01,02,11,12,22\}$. Successive functions are offset along the ordinate axis for clarity. The arrow highlights the emergence of the length-scale which corresponds to the first-sharp diffraction peak position in $S_{M M}(k)$. 